\title{
EVALUATION OF DATA APPLICABILITY FOR D-INSAR IN AREAS COVERED BY ABUNDANT VEGETATION
}

\author{
Peng Zhang ${ }^{1,2}$, Zheng Zhao, ${ }^{1,2}$ \\ ${ }^{1}$ Shandong University of Science and Technology, Qingdao, China - 1242195870@qq.com \\ ${ }^{2}$ Key Laboratory of Earth Observation and Geospatial Information Science of NASG, Chinese Academy of Surveying and Mapping, \\ Beijing, China
}

Commission III, ICWG III/IVb

KEY WORDS: Evaluation of Data Applicability, D-InSAR, Geologic Hazard, Areas Covered by Abundant Vegetation, Spatial Decorrelation, Interferometric Constraints

\begin{abstract}
:
In the past few years, the frequent geological disasters have caused enormous casualties and economic losses. Therefore, D-InSAR (differential interferometry synthetic aperture radar) has been widely used in early-warning and post disaster assessment. However, large area of decorrelation often occurs in the areas covered with abundant vegetation, which seriously affects the accuracy of surface deformation monitoring. In this paper, we analysed the effect of sensor parameters and external environment parameters on special decorrelation. Then Synthetic Aperture Radar (SAR) datasets acquired by X-band TerraSAR-X, Phased Array type L-band Synthetic Aperture Satellite-2 (ALOS-2), and C-band Sentinel-1 in Guizhou province were collected and analysed to generate the maps of coherence, which were used to evaluating the applicability of datasets of different wavelengths for D-InSAR in forest area. Finally, we found that datasets acquired by ALOS-2 had the best monitoring effect.
\end{abstract}

\section{INTRODUCTION}

Over the past couple of years, the geologic hazard occurred frequently in China. In 2017, more than 7000 geological disasters occurred, which caused 327 deaths and direct economic losses of 3 billion 540 million yuan. More than $85 \%$ of the geological hazards occurred in the mountainous areas covered by many vegetation in South and South China, where the natural conditions were changeable that the bench marks and GPS points in traditional survey technology were easily destroyed. At the same time, the complex terrain also made the field survey extremely difficult, greatly reducing the timeliness of disaster monitoring. Therefore, as an all-day and all-weather monitoring means of remote sensing, D-InSAR technology has become the better choice for the deformation monitoring (Dong, 2018; Zhao 2012).

Although the monitoring precision of the land surface deformation is enable to achieve centimeter level, even millimeter level, along the line of sight (LOS), it still has a lot of restrictions $(\mathrm{Ge}, 2013)$ : 1) The loss of coherence, or decorrelation, will result in the inability of the technology to correctly invert the changes in geophysical properties and surface deformation monitoring (Massonnet, 1998); 2) The change in phase gradient due to changes in surface deformation gradient ; 3) The phase delay due to atmospheric fluctuations.

For repeat-pass interferometry, the master and slave images must first meet the coherence condition, i.e. they should maintain high coherence in time and space. If the coherence is low or completely lost, the correct phase unwrapping result cannot be obtained, and the interference and differential interference results that can reflect the true deformation cannot be obtained. The factors that influence the decorrelation include temporal decorrelation, spatial decorrelation, imaging area geomorphology, geophysical activity, and data processing. Among them, spatial decorrelation is affected by two observation positions, which is closely related to the parameters of the selected data. This article quantitatively analyze the influence of several components (incident angle, wavelength, frequency bandwidth of LFM signals, etc.) related to sensor parameters on spatial decorrelation, and compares and analyzes the monitoring applicability of each sensor. Finally, the TerraSAR-X, COSMO-SkyMed, Sentinel-1, and ALOS-2 data in Guizhou were selected for verification.

\section{SPATIAL DECORRELATION}

Spatial de-correlation is the result of radar waves interacting with ground objects at different angles of incidence. Its mathematical definition can be expressed by the following formula:

$$
\gamma_{\text {spatial }}=\gamma_{\text {surface }} \cdot \gamma_{\text {volume }}
$$

Where $\gamma_{\text {surface }}=$ surface scattering decorrelation component

$\gamma_{\text {volume }}=$ volume scattering decorrelation component Surface scattering decorrelation: On the same reference plane, due to the difference in the viewing angles of the two SAR images, the ground reflection spectrum is not exactly the same, and the relative shift of the reflection spectrum of the two images results in incomplete agreement of the echo signals. Therefore, $\gamma_{\text {surface }}<1$.

The volume scattering decorrelation depends on the penetrating power of the electromagnetic wave, which is related to the wavelength of the electromagnetic wave and the penetrability and volume of the scatterer. In the vegetation coverage area (forest, farmland, etc.), $\gamma_{\text {volume }}$ dominates $\gamma_{\text {surface }}$ (Langley, 2007). 


\subsection{Surface scattering decorrelation}

At present, a large number of documents (Liu, 2016; Zhao, 2010) have described the geometric baseline error in detail, and the frequency-filtering technique can greatly reduce the geometric decorrelation caused by the baseline. However, in the monitoring of geologic hazards, the monitoring area is usually a mountainous terrain, and the local slope will also contribute to spatial de-correlation. Although multi-view filtering and other processing can effectively suppress the noise and reduce the error, the deformation information of the signal blind zone caused by the terrain features and the SAR system parameters cannot be recovered.

The model of surface scattering decorrelation related to slope and incident angle can be represented by the following formula:

$$
Y_{\text {surface }}=1-\frac{\sigma B_{\perp}}{\left.\lambda R B_{\text {in }} \mid \tan \left(\theta_{\mathrm{ins}}-\alpha\right)\right]}
$$

where ${ }^{C}=$ Light speed

$B_{\perp}=$ The length of effective baseline (vertical baseline)

$\lambda=$ Wavelength

$R=$ The target-to-satellite distance

$B_{w}=$ The frequency bandwidth of LFM signal

$\theta_{\text {ine }}=$ The incident angle

$\alpha=$ The slope angle

From equation (2), it can be seen that in the case of the same vertical baseline, different sensors have different resistance to surface scattering decorrelation caused by ${ }^{\alpha}$. Figure 1 shows the variation of surface scattering decorrelation for each sensor at different slope angles.

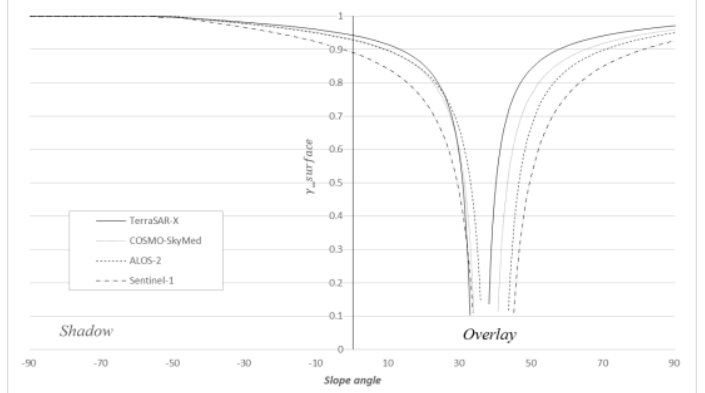

Figure 1 The relationship between surface scattering decorrelation and slope angle

Obviously, the coherence sharply decays when the slope angle approaches the incident angle, which means that for each sensor, there is a critical slope range determined by the sensor parameters and the vertical baseline. The formula for the critical slope range can be obtained from equation (2):

$$
\alpha_{\text {crit }}=\left[\theta_{\text {ine }}-\tan ^{-1}\left(\frac{c B_{\perp}}{\lambda R B_{w}}\right), \theta_{\text {ine }}+\tan ^{-1}\left(\frac{c B_{\perp}}{\lambda R B_{i v}}\right)\right]
$$

Researchers can use the a priori information (such as SRTM data) to calculate the slope of the study area, and then use the formula (3) to select the ideal sensor data.

\subsection{Volume scattering decorrelation}

According to literature (Askne, 2005), the volume scattering decorrelation model for vegetation coverage can be expressed as:

$$
Y_{\text {volume }}\left(B_{\perp}, h, \beta\right)=\frac{\beta}{\beta-j K} \cdot \frac{e^{-j h_{h}}-e^{-\beta h}}{1-e^{-\beta h}}
$$

where $K=4 \pi B_{\perp} /\left(\lambda R \sin \theta_{\text {inc }}\right)$

$\beta=$ The empirically defined coefficient, which can be considered to be related to some sort of two-way forest transmissivity.

$$
h=\text { Vegetation height }
$$

From equation (4), it can be seen that the volume scattering decorrelation is inseparable from the system parameters and ground parameters.

Taking Sentinel-1 as an example, assuming that $B_{\perp}=300 \mathrm{~m}$, $\beta=0.5 \mathrm{~dB} / \mathrm{m}, 1 \mathrm{~dB} / \mathrm{m}, 2 \mathrm{~dB} / \mathrm{m}$, the relationship between volume scattering correlation and vegetation height is shown in Figure 2.

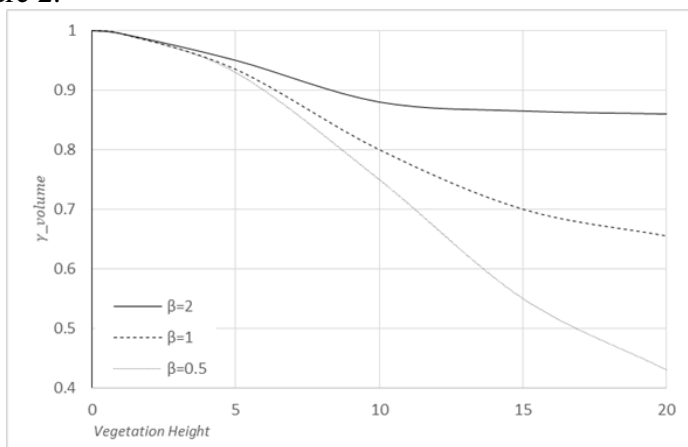

Figure 2 The relationship between volume scattering decorrelation and vegetation height (under different $\beta$ )

Assuming that $B_{\perp}=300 \mathrm{~m}$ and $\beta=1 \mathrm{~dB} / \mathrm{m}$, under different sensor parameters, the relationship between volume scatter decorrelation and vegetation height is shown in Figure 3.

It can be seen on the above two figures that: 1) With the increase of vegetation height, the volume scattering decorrelation decays continuously, and with the increase of the two-way forest transmissivity $(\beta)$, the decay rate of volume scattering decorrelation decreases; 2) In the same two-way forest transmissivity, the L-band sensor (ALOS-2) has the lowest sensitivity to vegetation height, followed by the C-band sensor (Sentinel-1), and the X-band sensors (COSMO-SkyMed and TerraSAR-X) are most sensitive. This result shows that the wavelength is dominant in the volume scattering decorrelation compared to other sensor parameters. 


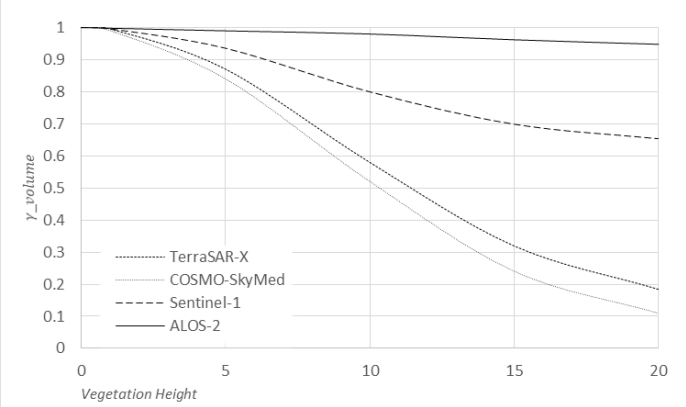

Figure 3 The relationship between volume scattering decorrelation and vegetation height (under different sensor parameters)

\section{DATA PROCESSING ANALYSIS}

This section uses four pairs of datasets, selected by TerraSAR-X, COSMO-SkyMed and Sentinel-1 in Guiyang City and ALOS-2 in Liupanshui City, for actual data experiments to verify what is described in Section 2

\subsection{Experimental Data and Area}

The experimental area was selected in Guiyang City, Guizhou Province and Liupanshui City, where the topography is fluctuating, the air humidity is high, the vegetation biomass is abundant, which causes serious decorrelation and provides favorable conditions for the analysis of interference restriction. Since the data coverage area of ALOS-2 does not overlap with the other three sensors, we chose two regions with similar natural conditions in the ALOS-2 coverage area and the overlap of the other three sensors, Region 1 and Rigion 2 as shown below (Figure 4).

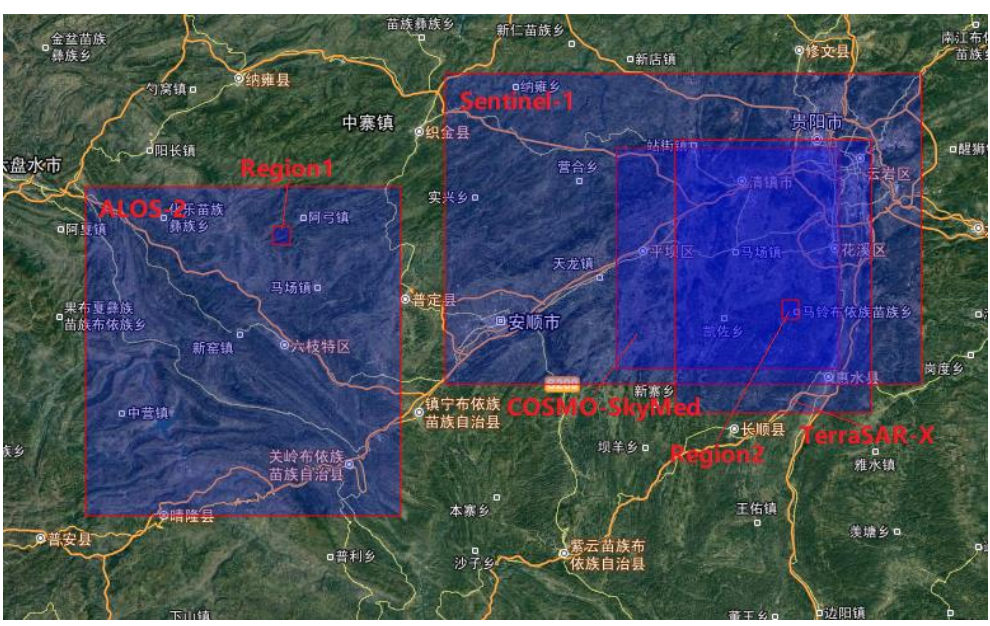

Figure 4 The coverage area of SAR data

Table 1 shows the detailed parameters of the selected data.

\begin{tabular}{|c|c|c|c|c|c|c|c|}
\hline Sensor & Selected date & Time-baseline(days) & Vertical-baseline(m) & Bandwidth(MHz) & Distance $(\mathrm{km})$ & Incident angle $\left(^{\circ}\right)$ & Wavelength $(\mathrm{cm})$ \\
\hline \multirow{2}{*}{ TerraSAR-X } & 20170728 & \multirow{2}{*}{22} & \multirow{2}{*}{208} & \multirow{2}{*}{150} & \multirow{2}{*}{613.7} & \multirow{2}{*}{35.3} & \multirow{2}{*}{2.4} \\
\hline & 20170819 & & & & & & \\
\hline \multirow[b]{2}{*}{ COSMO-SkyMed } & 20170803 & \multirow[b]{2}{*}{20} & \multirow[b]{2}{*}{400} & \multirow[b]{2}{*}{93} & \multirow[b]{2}{*}{767.7} & \multirow[b]{2}{*}{37.2} & \multirow[b]{2}{*}{2.4} \\
\hline & 20170823 & & & & & & \\
\hline \multirow{2}{*}{ Sentinel-1 } & 20170725 & \multirow{2}{*}{24} & \multirow{2}{*}{93} & \multirow{2}{*}{48.3} & \multirow{2}{*}{875.1} & \multirow{2}{*}{39.3} & \multirow{2}{*}{5.66} \\
\hline & 20170818 & & & & & & \\
\hline \multirow{2}{*}{ ALOS-2 } & 20170611 & \multirow{2}{*}{46} & \multirow{2}{*}{183} & \multirow{2}{*}{79.4} & \multirow{2}{*}{799.4} & \multirow{2}{*}{39.67} & \multirow{2}{*}{23.6} \\
\hline & 20170806 & & & & & & \\
\hline
\end{tabular}

Table 1 The parameters of SAR data

\subsection{Results and Analysis}

The SRTM-DEM, with resolution of $30 \mathrm{~m}$, was selected to perform slope inversion for Region 1 and Region 2. The results are shown in Figure 5(a) and (b).
After extracting the coherence coefficients of the four groups of interference pairs, we have obtained the following results in Figure 6 (a) (d).

For a more intuitive analysis, we conducted a statistical analysis of the four results, which are shown in Figure 7 (a) (d) and Table 2 . 


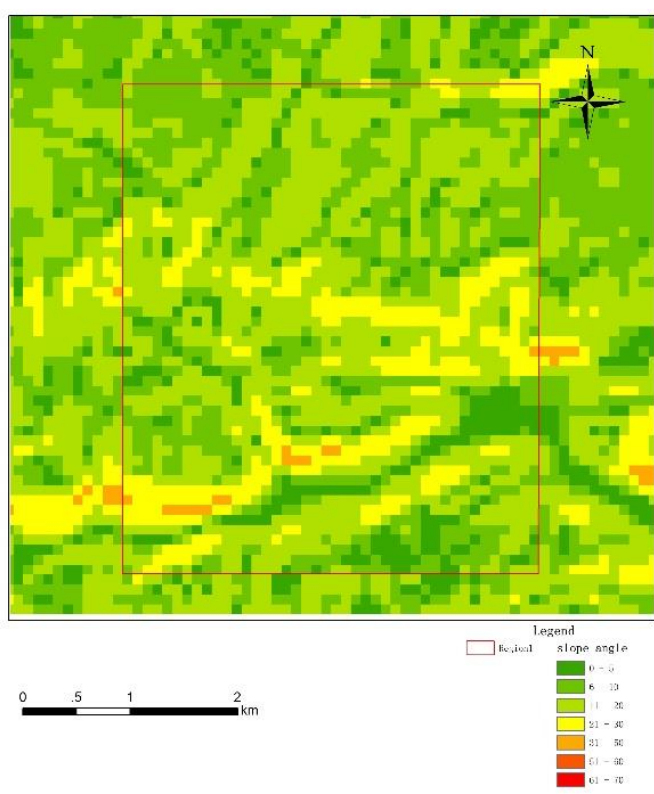

(a)

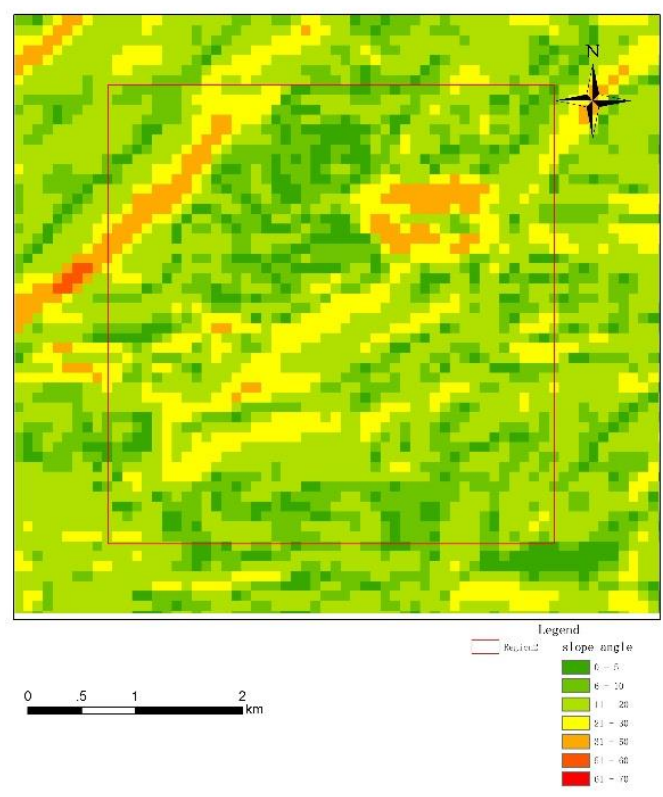

(b)

Figure 5 Slope in Region 1 \& Region 2

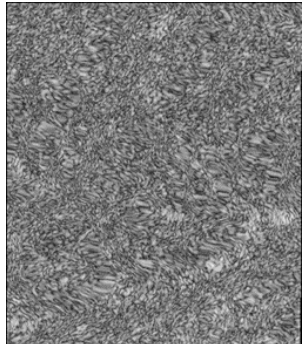

(a) TerraSAR-X

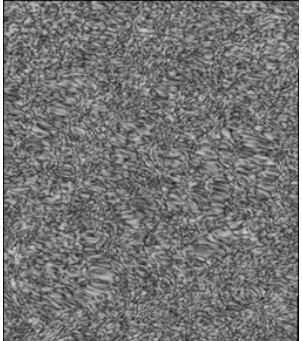

(b)COSMO-SkyMed

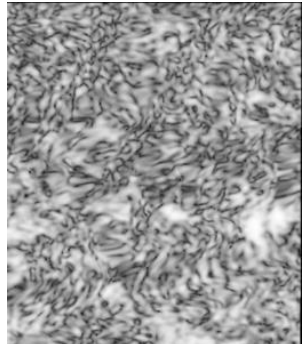

(c)Sentinel-1

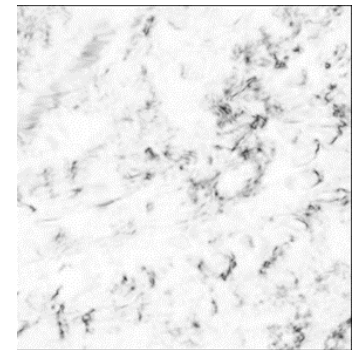

(d)ALOS-2

Figure 6 Coherence value of the four groups of interference pairs

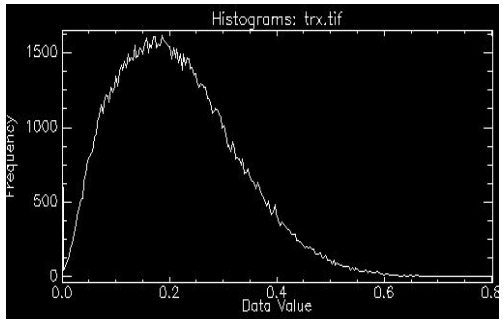

a TerraSAR-X

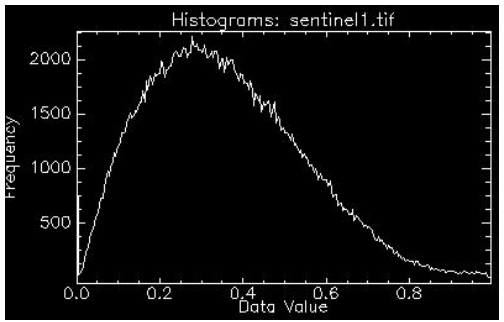

c Sentinel1

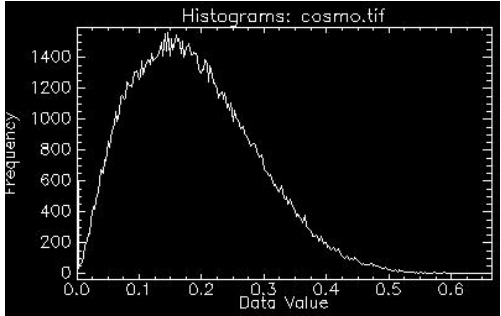

b COSMO-SkyMed

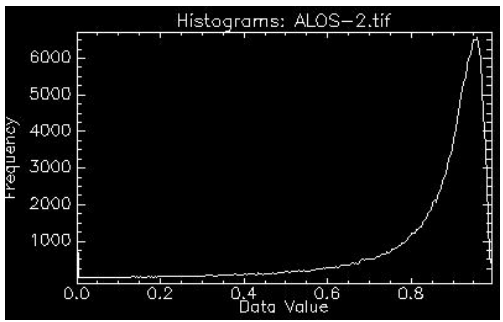

d ALOS-2

Figure 7 Histogram of the four groups of interference pairs 


\begin{tabular}{|c|c|c|c|c|}
\hline Data & Min & Max & Mean & StDev \\
\hline TerraSAR-X & 0 & 0.801715 & 0.214061 & 0.113214 \\
\hline Sentinel1 & 0 & 0.995223 & 0.355915 & 0.183840 \\
\hline COSMO-SkyMed & 0 & 0.664783 & 0.188541 & 0.098797 \\
\hline ALOS-2 & 0 & 0.996479 & 0.857580 & 0.152360 \\
\hline
\end{tabular}

Table 2 The statistical results of the four groups of interference pairs

As can be seen from Figure 6, the overall coherence coefficient of ALOS-2 is high and continuous; the coherence coefficient of Sentinel1 is the next highest, and the continuity is worse than ALOS-2, and there are many low coherence gaps; TerraSAR-X and COSMO-SkyMed have the lowest coherence coefficients and are very fragmented. From Figure 7 and Table 2, it can be seen that :

$$
\gamma_{\text {ALOS-2 }}>\gamma_{\text {Sentinel1 }}>\gamma_{\text {TerraSAR-X }} \approx \gamma_{\text {COSMo-SkyMed }}
$$

The content described in Section 2.2 is proudly proved.

\section{CONCLUTION}

This paper analyzes the applicability of the current mainstream SAR systems in vegetation coverage from two aspects: surface scattering decorrelation and volume scattering decorrelation. Through comparison and analysis of data, the SAR system, ALOS-2, with better coherence coefficient can obtain better SAR Interference results in topographically variable vegetation coverage area. This is of great significance for obtaining more accurate geo-hazard deformation monitoring results.

\section{ACKNOWLEDGMENT}

This work was supported in part by the basic plan of science and technology of Surveying and mapping (No. 2018KJ0103).

\section{REFERENCES}

Dong J., Zhang L., Li M.H., et al., 2018. Measuring precursory movements of the recent Xinmo landslide in Mao County, China with Sentinel-1 and ALOS-2 PALSAR-2 datasets. Landslides, 15(1): 135-144.

Zhao C.Y., Lu Z., Zhang Q., et al., 2012. Large-area landslide detection and monitoring with ALOS/PALSAR imagery data over Northern California and Southern Oregon, USA. Remote Sensing of Environment, 124: 348-359.

Ge D.Q., 2013. Research on the key techniques of SAR interferometry for regional land subsidence monitoring. A Dissertation Submitted to China University of Geoscience for Doctoral Degree.

Massonnet D., Feigl K.L., 1998. Radar interferometry and its application to changes in the Earth's surface. Reviews of Geophysics, 36(4): 441-500.

Langley K., Hamran S.E., Hogda K.A., et al., 2007. Use of Cband ground penetrating radar to determine backscatter sources within glaciers. IEEE Transactions on Geoscience and Remote Sensing, 45(5): 1236-1246.
Liu G., Hanssen R.F., Guo H.D., et al., 2016. Nonlinear model for InSAR baseline error. IEEE Transactions on Geoscience and Remote Sensing, 54(9): 5341-5351.

Zhao H.L., Fan J.H., Guo X.F., 2010. A method for InSAR baseline refinement and its application. Second IITA International Conference on Geoscience and Remote Sensing, 2: 161-164.

Askne J., Santoro M., Smith G., et al., 2003. Multitemporal repeat-pass SAR interferometry of boreal forests. IEEE Transactions on Geoscience and Remote Sensing, 41(7): 15401550 . 